\title{
Metal-semiconductor nanoheterostructures with an AIGaN quantum well and in-situ formed surface Al nanoislands
}

(C) E.A. Evropeytsev, A.N. Semenov, D.V. Nechaev, V.N. Jmerik, V.Kh. Kaibyshev,

S.I. Troshkov, P.N. Brunkov, A.A. Usikova, S.V. Ivanov, A.A. Toropov

loffe Institute,

194021 St. Petersburg, Russia

E-mail: evropeitsev@beam.ioffe.ru

We report on fabrication and studies of composite heterostuctures consisting of an $\mathrm{Al}_{0.55} \mathrm{Ga}_{0.45} \mathrm{~N} / \mathrm{Al}_{0.8} \mathrm{Ga}_{0.2} \mathrm{~N}$ quantum well and surface $\mathrm{Al}$ nanoislands, grown by plasma-assisted molecular-beam epitaxy on $c$-sapphire substrates. The influence of a substrate temperature varied between 320 and $700^{\circ} \mathrm{C}$ on the size and density of the deposited $\mathrm{Al}$ nanoislands is evaluated. The effect of $\mathrm{Al}$ nanoislands on decay kinetics of the quantum well middle-ultraviolet photoluminescence has been investigated by time resolved photoluminescence. The samples with the maximum density of $\mathrm{Al}$ nanoislands of $10^{8} \mathrm{~cm}^{-2}$ and lateral dimensions in the range of $100-500 \mathrm{~nm}$ demonstrated shortening of the photoluminescence lifetime, induced by interaction of the emitting quantum well and the plasmonic metal particles.

\section{Acknowledgements}

This work has been supported in part by RFBR \# 15-02-05206. The development of the MBE technology and samples growth was supported by the Russian Science Foundation (project\#14-22-00107). 